\title{
Circumventing the relative degree condition in sliding mode design
}

\author{
Christopher Edwards ${ }^{1}$, Thierry Floquet ${ }^{2}$ and Sarah Spurgeon ${ }^{1}$ \\ 1 Control and Instrumentation Research Group, University of Leicester, UK \\ chris.edwards@le.ac.uk, eon@le.ac.uk \\ 2 LAGIS UMR CNRS 8146, Ecole Centrale de Lille, BP 48, Cité Scientifique, \\ 59651 Villeneuve-d'Ascq, France Thierry.Floquet@ec-lille.fr
}

Summary. Classical sliding mode design approaches assume the transfer function matrix between the driving signal and the measured output of interest must be minimum phase and relative degree one. For the control case, the driving signal will be the control input and for the observer problem the driving signal is likely to be an unknown input which the observer seeks to reconstruct. This chapter demonstrates that the relative degree condition can be weakened if the nominal linear system used for the controller or observer design is combined with sliding mode exact differentiators to essentially generate additional independent output signals from the available measurements. It is shown that the transmission zeros of the original plant appear directly in the reduced order sliding mode dynamics relating to the augmented system in both cases. In the case of output feedback sliding mode control design for MIMO systems of any relative degree, a super twisting control algorithm is shown to provide robust control performance. Nonlinear simulation results for a ninth order nonlinear description of a web transport system, which does not satisfy the usual relative degree one condition, are used to demonstrate both the control design approach and the design of an unknown input observer.

Key words: sliding mode; sliding mode differentiators; output feedback; observer design

\section{Introduction}

A continuous time sliding mode is generated by means of discontinuities in the applied injection signals, about a surface in the state space $[17,33,40]$. The discontinuity surface (usually known as the sliding surface) is attained from any initial condition ideally in a finite time interval. Provided the injection signals are designed appropriately, the motion when constrained to the surface (the sliding mode) is completely insensitive to so-called matched uncertainties, i.e. uncertainties that lie within the range space of the matrix distributing the injection signals. Much early work in this area related to control problems and 
assumed all of the states were available for use both in the switching function evaluation and also by the control law. For practical application however, the case when only limited state information is available is of interest.

A number of algorithms have been developed for robust stabilization of uncertain systems which are based on sliding surfaces and output feedback control schemes [16], [45]. In [45] a geometric condition is developed to guarantee the existence of the sliding surface and the stability of the reduced order sliding motion. Edwards and Spurgeon derived an algorithm [16], [17] which is convenient for practical use. In both these results, it is required that the disturbance considered is matched, i.e. acts in the channels of the inputs. In many cases, however, the disturbance suffered by practical systems does not act in the input channel. Unlike the matched case, any mismatched disturbance impinges on the sliding mode dynamics and affects the behaviour of the sliding mode directly [44]. Based on the work in [45], some dynamic output feedback control schemes have been proposed [30], [35]. Unfortunately, in all the above output feedback sliding mode control schemes, it is an a priori requirement that the system under consideration is minimum phase and relative degree one.

The concept of sliding mode control has been extended to the problem of state estimation by an observer, for linear systems [40], uncertain linear systems $[15,42]$ and nonlinear systems $[1,13,36]$. Using the same design principles as for variable structure control, the observer trajectories are constrained to evolve after a finite time on a suitable sliding manifold by the use of a discontinuous output injection signal (the sliding manifold is usually given by the difference between the observer and the system output). Subsequently the sliding motion provides an estimate (asymptotically or in finite time) of the system states. Sliding mode observers have been shown to be efficient in many applications, such as in robotics [4, 27], electrical engineering [11, 21, 41], and fault detection $[20,22]$. The necessary and sufficient conditions for the existence of a 'classical' sliding mode observer ${ }^{3}$ as described in $[15,42]$ is that the transfer function matrix between the unmeasurable inputs (or disturbances) and the measured outputs must be minimum phase and relative degree one.

This chapter shows how it is possible to broaden the class of systems for which both sliding mode output feedback controllers and observers can be designed. It is shown that the relative degree condition can be weakened in both cases if the sliding mode controller or observer is combined with sliding mode exact differentiators to generate additional independent output signals from the available measurements. The work has its roots in the contribution of [5] where an output feedback sliding mode controller for MIMO systems of any relative degree is considered. It is assumed that the input explicitly appears first in the $r$-th time derivatives of each of the $p$ outputs of the system. In [5], the authors take $r$ derivatives of each measured output and introduce an integral sign function control, whereby effectively the control is designed to

\footnotetext{
${ }^{3}$ A precise observer description will be given later in the Chapter.
} 
determine the derivative of the actual applied input signal. Here the number of outputs requiring differentiation is minimized and a robust sliding mode differentiator is presented as the means to construct the extended output signal. It is shown that the transmission zeros of the triple used to design the controller or observer appear directly in the reduced order sliding mode dynamics. For the static output feedback control problem, a twisting control algorithm is shown to provide robust control performance. For the sliding mode observer design problem, a classical first order observer is described which estimates the system states and any unknown inputs. Nonlinear simulation results for a ninth order nonlinear description of a web-transport system, which does not satisfy the usual relative degree conditions required for sliding mode output feedback controller or observer design, are used to demonstrate the efficacy of the approach.

\section{Motivation and General Problem Statement}

Consider an uncertain dynamical system of the form

$$
\begin{aligned}
\dot{x}(t) & =A x(t)+B u(t)+f(t, x, u) \\
y & =\left[y_{1} \cdots y_{p}\right]^{T}=C x, y_{i}=C_{i} x
\end{aligned}
$$

where $x \in \mathbb{R}^{n}, u \in \mathbb{R}^{m}$ and $y \in \mathbb{R}^{p}$ with $m \leq p<n$. It is assumed that the system (1) is Bounded Input Bounded State (BIBS), that the nominal linear system $(A, B, C)$ is known with $(A, B)$ controllable and that the input and output matrices $B$ and $C$ are both full rank. The function $f: \mathbb{R}_{+} \times \mathbb{R}^{n} \times \mathbb{R}^{m} \rightarrow$ $\mathbb{R}^{n}$ represents system nonlinearities, uncertainties, disturbances or any other unknown input present in the system. It is assumed to be bounded as well as having a bounded first time derivative: i.e. there exists a smooth vector field $\xi(t, x, u) \in \mathbb{R}^{q}$, a known constant matrix $D \in \mathbb{R}^{n \times q}$ and some known constants $K$ and $K^{\prime}$ such that:

$$
f(t, x, u)=D \xi(t, x, u), \quad\|\xi(t, x, u)\|<K, \quad\|\dot{\xi}(t, x, u)\|<K^{\prime}
$$

In the case of the development of a control law based on output measurements only, all uncertainties have been assumed to be matched, so that $D=B$ (and as a consequence, $q=m$ ). Then, the problem is to induce an ideal sliding motion on the surface

$$
S=\left\{x \in \mathbb{R}^{n}: F C x=0\right\}
$$

for some selected matrix $F \in \mathbb{R}^{m \times p}$. It is well known that for a unique equivalent control to exist, the matrix $F C B \in \mathbb{R}^{m \times m}$ must have full rank. As

$$
\operatorname{rank}(F C B) \leq \min \{\operatorname{rank}(F), \operatorname{rank}(C B)\}
$$


it follows that both $F$ and $C B$ must have full rank. As $F$ is a design parameter, it can be chosen to be full rank. A necessary condition for $F C B$ to be full rank, and thus for solvability of the output feedback sliding mode design problem, thus becomes that $C B$ must have rank $m$. If this rank condition holds and any invariant zeros of the triple $(A, B, C)$ lie in $\mathbb{C}_{-}$, then the existence of a matrix $F$ defining the surface (3) which provides a stable sliding motion with a unique equivalent control is determined from the stabilizability by output feedback of a specific, well-defined subsystem of the plant [17]. Here, the first aim is to extend the existing results so that a sliding mode controller based on output measurements can be designed for the system (1-2) when $\operatorname{rank}(C B)$ is strictly less than $m$.

The second aim is to develop a sliding mode observer for the system (1), when driven by unknown inputs $\xi$. Without loss of generality, it can be assumed that $\operatorname{rank}(D)=q$. Consider a sliding mode observer of the general form

$$
\dot{\hat{x}}=A \hat{x}+B u+G_{l}(y-C \hat{x})+G_{n} v_{c}
$$

where $G_{l}$ and $G_{n}$ are design gains and $v_{c}$ is an injection signal which depends on the output estimation error in such a way that a sliding motion in the state estimation error space is induced in finite time. The objective is to ensure the state estimation error $e=x-\hat{x}$ is asymptotically stable and independent of the unknown signal $\xi$ during the sliding motion. As argued in [17] necessary and sufficient conditions to solve this problem are: the invariant zeros of $\{A, D, C\}$ lie in $\mathbb{C}_{-}$and

$$
\operatorname{rank}(C D)=\operatorname{rank}(D)=q .
$$

In this chapter, the existing results are extended so that a sliding mode observer based on output measurements can be designed for the system (1-2) when $\operatorname{rank}(C D)$ is strictly less than $q$.

The next section will explore an output extension approach to circumvent the relative degree condition for both sliding mode controller and observer problems.

\section{Generation of the extended output}

Introduce the notion of relative degree $\mu_{j} \in \mathbb{N}^{*}, 1 \leq j \leq p$ of the system with respect to the output $y_{j}$, that is to say the number of times the output $y_{j}$ must be differentiated in order to have the unknown input $\xi$ explicitly appear. Thus, $\mu_{j}$ is defined as follows:

$$
\begin{aligned}
C_{j} A^{k} D & =0, \text { for all } k<\mu_{j}-1 \\
C_{j} A^{\mu_{j}-1} D & \neq 0 .
\end{aligned}
$$

Without loss of generality, it is assumed that $\mu_{1} \leq \ldots \leq \mu_{p}$. The following assumptions are made: 
- the invariant zeros of $\{A, D, C\}$ lie in $\mathbb{C}_{-}$

- there exists a full rank matrix

$$
\tilde{C}=\left[\begin{array}{c}
C_{1} \\
\vdots \\
C_{1} A^{\mu_{\alpha_{1}-1}} \\
\vdots \\
C_{p} \\
\vdots \\
C_{p} A^{\mu_{\alpha_{p}}-1}
\end{array}\right]
$$

where the integers $1 \leq \mu_{\alpha_{i}} \leq \mu_{i}$ are such that $\operatorname{rank}(\tilde{C} D)=\operatorname{rank}(D)$ and the $\mu_{\alpha_{i}}$ are chosen such that $\sum_{i=1}^{p} \mu_{\alpha_{i}} \triangleq \tilde{p}$ is minimal.

The following lemma will demonstrate that the invariant zeros of the triple $\{A, D, C\}$ and the newly created triple with additional (derivative) outputs $\{A, D, \tilde{C}\}$ are identical.

Lemma 1. The invariant zeros of the triples $\{A, D, C\}$ and $\{A, D, \tilde{C}\}$ are identical.

Proof: Suppose $s_{0} \in \mathbb{C}$ is an invariant zero of $\{A, D, \tilde{C}\}$. Consequently $\left.\tilde{P}(s)\right|_{s=s_{0}}$ loses normal rank, where $\tilde{P}(s)$ is Rosenbrock's system matrix defined by

$$
\tilde{P}(s):=\left[\begin{array}{cc}
s I-A & D \\
\tilde{C} & 0
\end{array}\right]
$$

Since by assumption $p \geq m$, this implies $\tilde{P}(s)$ loses column rank and therefore there exist non-zero vectors $\eta_{1}$ and $\eta_{2}$ such that

$$
\begin{aligned}
\left(s_{0} I-A\right) \eta_{1}+D \eta_{2} & =0 \\
\tilde{C} \eta_{1} & =0
\end{aligned}
$$

From the definition of $\tilde{C}, \tilde{C} \eta_{1}=0 \Rightarrow C \eta_{1}=0$. Consequently

$$
\begin{aligned}
\left(s_{0} I-A\right) \eta_{1}+D \eta_{2} & =0 \\
C \eta_{1} & =0
\end{aligned}
$$

and so $\left.P(s)\right|_{s=s_{0}}$ loses column rank where

$$
P(s):=\left[\begin{array}{cc}
s I-A & D \\
C & 0
\end{array}\right]
$$

is Rosenbrock's System Matrix for the triple $\{A, D, C\}$. Therefore any invariant zero of $\{A, D, \tilde{C}\}$ is an invariant zero of $\{A, D, C\}$. 
Now suppose $s_{0} \in \mathbb{C}$ is an invariant zero of $\{A, D, C\}$. This implies the existence of non-zero vectors $\eta_{1}$ and $\eta_{2}$ such that

$$
\begin{aligned}
\left(s_{0} I-A\right) \eta_{1}+D \eta_{2} & =0 \\
C \eta_{1} & =0
\end{aligned}
$$

The first (sub) equation of (9) implies $C_{1} \eta_{1}=0$. Suppose $\mu_{\alpha_{1}}>1$. Then multiplying (8) by $C_{1}$ gives

$$
s_{0} \underbrace{C_{1} \eta_{1}}_{=0}-C_{1} A \eta_{1}+\underbrace{C_{1} D}_{=0} \eta_{2}=0
$$

which implies $C_{1} A \eta_{1}=0$. By an inductive argument it follows that $C_{1} A^{k} \eta_{1}=$ 0 for $k \leq \mu_{\alpha_{1}}-1$. Repeating this analysis for $C_{2}$ up to $C_{p}$ it follows

$$
C_{j} A^{k} \eta_{1}=0 \quad \text { for } k \leq \mu_{\alpha_{j}}-1, \quad j=1 \ldots p
$$

and therefore

$$
\tilde{C} \eta_{1}=0
$$

Consequently, from (10) and (8), $s_{0}$ is an invariant zero of the triple $\{A, D, \tilde{C}\}$ and the lemma is proved.

Implementation of the differentiators required to construct the extended output signals will now be discussed. The aim is to recover in finite time knowledge of the partially measured output vector generated by $\tilde{C} x$. The problem can be seen as one of designing an observer for a system that can be put in a so-called canonical triangular observable form. Most of the sliding mode observer designs for such a form are based on a step-by-step procedure using successive filtered values of the so-called equivalent output injections obtained from recursive first order sliding mode observers (see e.g. [1, 12, $13,25,32,43])$. However, the approximation of the equivalent injections by low pass filters at each step will typically introduce some delays that lead to inaccurate estimates or to instability for high order systems. To overcome this problem, the discontinuous first order sliding mode output injection is replaced by a continuous second order sliding mode one.

Define the following sliding mode observer based on a so-called step-bystep observer:

$$
\left\{\begin{array}{l}
\dot{y}_{i}^{1}=\nu\left(y_{i}-y_{i}^{1}\right)+C_{i} B u \\
\dot{y}_{i}^{2}=E_{1} \nu\left(\tilde{y}_{i}^{2}-y_{i}^{2}\right)+C_{i} A B u \\
\vdots \\
\dot{y}_{i}^{\mu_{\alpha_{i}}-1}=E_{\mu_{\alpha_{i}}-2} \nu\left(\tilde{y}_{i}^{\mu_{\alpha_{i}}-1}-y_{i}^{\mu_{\alpha_{i}}-1}\right)+C_{i} A^{\mu_{\alpha_{i}}-2} B u
\end{array}\right.
$$

for $1 \leq i \leq p$, with

$$
\begin{aligned}
& \tilde{y}_{i}^{1}:=y_{i} \\
& \tilde{y}_{i}^{j}:=\nu\left(\tilde{y}_{i}^{j-1}-y_{i}^{j-1}\right), 2 \leq j \leq \mu_{\alpha_{i}}-1
\end{aligned}
$$


where the continuous output error injection $\nu(\cdot)$ is given by the so-called super twisting algorithm [31]:

$$
\left\{\begin{array}{l}
\nu(s)=\varphi(s)+\lambda_{s}|s|^{\frac{1}{2}} \operatorname{sign}(s) \\
\dot{\varphi}(s)=\alpha_{s} \operatorname{sign}(s) \\
\lambda_{s}, \alpha_{s}>0
\end{array} .\right.
$$

For $j=1, \ldots, \mu_{\alpha_{i}}-2$, the scalar functions $E_{i}$ are defined as

$$
E_{j}=1 \text { if }\left|\tilde{y}_{i}^{k}-y_{i}^{k}\right| \leq \varepsilon, \text { for all } k \leq j \text { else } E_{i}=0
$$

where $\varepsilon$ is a small positive constant. This is an anti-peaking structure [37]. As argued in [1], with this particular function, the manifolds are reached one by one. At each step, a sub-dynamic of dimension one is obtained and consequently no peaking phenomena appear. Define the augmented output estimation error $e_{y}=\tilde{C} x-\bar{y}$, with

$$
\begin{aligned}
e_{y} & \triangleq\left[e_{1}^{1}, \ldots, e_{1}^{\mu_{\alpha_{1}}-1}, \ldots, e_{p}^{1}, \ldots, e_{p}^{\mu_{\alpha_{p}}-1}\right]^{T} \\
\bar{y} & =\left[y_{1}^{1}, \ldots, y_{1}^{\mu_{\alpha_{1}}-1}, \ldots, y_{p}^{1}, \ldots, y_{p}^{\mu_{\alpha_{p}}-1}\right]^{T}
\end{aligned}
$$

then it is straightforward to show that:

$$
\left\{\begin{array}{l}
\dot{e}_{i}^{1}=C_{i}(A x+B u+D \xi)-\nu\left(y_{i}-y_{i}^{1}\right)-C_{i} B u=C_{i} A x-\nu\left(y_{i}-y_{i}^{1}\right) \\
\dot{e}_{i}^{2}=C_{i} A^{2} x-E_{1} \nu\left(\tilde{y}_{i}^{2}-y_{i}^{2}\right) \\
\vdots \\
\dot{e}_{i}^{\mu_{\alpha_{i}}-1}=C_{i} A^{\mu_{\alpha_{i}}-1} x-E_{\mu_{\alpha_{i}}-2} \nu\left(\tilde{y}_{i}^{\mu_{\alpha_{i}}-1}-y_{i}^{\mu_{\alpha_{i}}-1}\right)
\end{array}\right.
$$

$1 \leq i \leq p$. Since (1) is BIBS and $f, \dot{f}$ are bounded, it can be shown (see [23] and [34]) that, with suitable gains in the output injections $\nu$, a sliding mode appears in finite time on the manifolds $e_{i}^{j}=\dot{e}_{i}^{j}=0,1 \leq i \leq p, 1 \leq j \leq \mu_{\alpha_{i}}-1$. Thus, the following equations hold after a finite time $T$ :

$$
\begin{aligned}
& \nu\left(y_{i}-y_{i}^{1}\right)=C_{i} A x \\
& \nu\left(\tilde{y}_{i}^{2}-y_{i}^{2}\right)=C_{i} A^{2} x \\
& \vdots \\
& \nu\left(\tilde{y}_{i}^{\mu_{\alpha_{i}}-1}-y_{i}^{\mu_{\alpha_{i}}-1}\right)=C_{i} A^{\mu_{\alpha_{i}}-1} x
\end{aligned}
$$

for $1 \leq i \leq p$, and 


$$
\tilde{y} \triangleq\left[\begin{array}{c}
y_{1} \\
\nu\left(y_{1}-y_{1}^{1}\right) \\
\vdots \\
\nu\left(\tilde{y}_{1}^{\mu_{\alpha_{1}}-1}-y_{1}^{\mu_{\alpha_{1}}-1}\right) \\
\vdots \\
y_{p} \\
\vdots \\
\nu\left(\tilde{y}_{p}^{\mu_{\alpha_{p}}-1}-y_{p}^{\mu_{\alpha_{p}}-1}\right)
\end{array}\right]=\tilde{C} x .
$$

\section{The output feedback sliding mode control law}

The triple $(A, B, \tilde{C})$ in (1) and (7) is now considered and a static output feedback controller developed.

\subsection{Solution of the Existence Problem}

This subsection will present a constructive analysis determining when and how the sliding surface parameter $F$ can be constructed assuming the extended outputs are available. It is convenient to introduce, without loss of generality, a coordinate transformation to the usual regular form, making the final $\tilde{p}$ states of the system depend directly on the extended outputs [17]:

$$
A=\left[\begin{array}{ll}
A_{11} & A_{12} \\
A_{21} & A_{22}
\end{array}\right] \quad B=\left[\begin{array}{c}
0 \\
B_{2}
\end{array}\right] \quad \tilde{C}=\left[\begin{array}{ll}
0 & T
\end{array}\right]
$$

where $T \in \mathbb{R}^{\tilde{p} \times \tilde{p}}$ is an orthogonal matrix, $A_{11} \in \mathbb{R}^{(n-m) \times(n-m)}$ and the remaining sub-blocks in the system matrix are partitioned accordingly. Define a corresponding switching surface parameter by $\tilde{F} \in \mathbb{R}^{m \times \tilde{p}}$. Let

$$
\begin{aligned}
& \stackrel{\tilde{p}-m}{\leftrightarrow} \stackrel{m}{\leftrightarrow} \\
& {\left[\begin{array}{ll}
F_{1} & F_{2}
\end{array}\right]=\tilde{F} T}
\end{aligned}
$$

where $T$ is the matrix from equation (16). As a result

$$
\tilde{F} \tilde{C}=\left[\begin{array}{ll}
F_{1} C_{1} & F_{2}
\end{array}\right]
$$

where

$$
C_{1}=\left[0_{(\tilde{p}-m) \times(n-\tilde{p})} I_{(\tilde{p}-m)}\right]
$$

Therefore $\tilde{F} \tilde{C} B=F_{2} B_{2}$ and the square matrix $F_{2}$ is nonsingular. The canonical form in (16) is a special case of the regular form normally used in sliding mode controller design, and the reduced-order sliding motion is governed by a free motion with system matrix 


$$
A_{11}^{s}=A_{11}-A_{12} F_{2}^{-1} F_{1} C_{1}
$$

which must therefore be stable. If $K \in \mathbb{R}^{m \times(\tilde{p}-m)}$ is defined as $K=F_{2}^{-1} F_{1}$ then

$$
A_{11}^{s}=A_{11}-A_{12} K C_{1}
$$

and the problem of hyperplane design is equivalent to a static output feedback problem for the system $\left(A_{11}, A_{12}, C_{1}\right)$. In order to utilize the existing literature, it is necessary that the pair $\left(A_{11}, A_{12}\right)$ is controllable and $\left(A_{11}, C_{1}\right)$ is observable. The former is ensured as $(A, B)$ is controllable. The observability of $\left(A_{11}, C_{1}\right)$, is not so straightforward, but can be investigated by considering the canonical form below.

Lemma 2. Let $(A, B, \tilde{C})$ be a linear system with $\tilde{p}>m$ and $\operatorname{rank}(\tilde{C} B)=m$. Then a change of coordinates exists so that the system triple with respect to the new coordinates has the following structure:

- The system matrix can be written as

$$
A=\left[\begin{array}{ll}
A_{11} & A_{12} \\
A_{21} & A_{22}
\end{array}\right]
$$

where $A_{11} \in \mathbb{R}^{(n-m) \times(n-m)}$ and the sub-block $A_{11}$ when partitioned has the structure

$$
A_{11}=\left[\begin{array}{cc|c}
A_{11}^{o} & A_{12}^{o} & A_{12}^{m} \\
0 & A_{22}^{o} & \\
\hline 0 & A_{21}^{o} & A_{22}^{m}
\end{array}\right]
$$

where $A_{11}^{o} \in \mathbb{R}^{r \times r}, A_{22}^{o} \in \mathbb{R}^{(n-\tilde{p}-r) \times(n-\tilde{p}-r)}$ and $A_{21}^{o} \in \mathbb{R}^{(\tilde{p}-m) \times(n-\tilde{p}-r)}$ for some $r \geq 0$ and the pair $\left(A_{22}^{o}, A_{21}^{o}\right)$ is completely observable.

- The input distribution matrix $B$ and the output distribution matrix $\tilde{C}$ have the structure in (16).

For a proof and a constructive algorithm to obtain this canonical form see [16].

In the case where $r>0$, the intention is to construct a new system $\left(\tilde{A}_{11}, \tilde{B}_{1}, \tilde{C}_{1}\right)$ which is both controllable and observable with the property that

$$
\lambda\left(A_{11}^{s}\right)=\lambda\left(A_{11}^{o}\right) \cup \lambda\left(\tilde{A}_{11}-\tilde{B}_{1} K \tilde{C}_{1}\right) .
$$

As in [16], partition the matrices $A_{12}$ and $A_{12}^{m}$ as

$$
A_{12}=\left[\begin{array}{l}
A_{121} \\
A_{122}
\end{array}\right] \quad \text { and } \quad A_{12}^{m}=\left[\begin{array}{l}
A_{121}^{m} \\
A_{122}^{m}
\end{array}\right]
$$

where $A_{122} \in \mathbb{R}^{(n-m-r) \times m}$ and $A_{122}^{m} \in \mathbb{R}^{(n-\tilde{p}-r) \times(\tilde{p}-m)}$ and form a new subsystem $\left(\tilde{A}_{11}, A_{122}, \tilde{C}_{1}\right)$ where 


$$
\begin{aligned}
\tilde{A}_{11} & =\left[\begin{array}{ll}
A_{22}^{o} & A_{122}^{m} \\
A_{21}^{o} & A_{22}^{m}
\end{array}\right] \\
\tilde{C}_{1} & =\left[\begin{array}{ll}
0_{(\tilde{p}-m) \times(n-\tilde{p}-r)} & I_{(\tilde{p}-m)}
\end{array}\right]
\end{aligned}
$$

It follows that the spectrum of $A_{11}^{s}$ decomposes as

$$
\lambda\left(A_{11}-A_{12} K C_{1}\right)=\lambda\left(A_{11}^{o}\right) \cup \lambda\left(\tilde{A}_{11}-A_{122} K \tilde{C}_{1}\right)
$$

Lemma 3. [16] The spectrum of $A_{11}^{o}$ represents the invariant zeros of $(A, B, \tilde{C})$ which have been shown to be the invariant zeros of the original system triple $(A, B, C)$.

For a stable sliding motion, the invariant zeros of the system $(A, B, C)$ must lie in the open left-half plane and the triple $\left(\tilde{A}_{11}, A_{122}, \tilde{C}_{1}\right)$ must be stabilizable with respect to output feedback. The matrix $A_{122}$ is not necessarily full rank. Suppose $\operatorname{rank}\left(A_{122}\right)=m^{\prime}$ then, as in [16], it is possible to construct a matrix of elementary column operations $T_{m^{\prime}} \in \mathbb{R}^{m \times m}$ such that

$$
A_{122} T_{m^{\prime}}=\left[\tilde{B}_{1} 0\right]
$$

where $\tilde{B}_{1} \in \mathbb{R}^{(n-m-r) \times m^{\prime}}$ and is of full rank. If $K_{m^{\prime}}=T_{m^{\prime}}^{-1} K$ and $K_{m^{\prime}}$ is partitioned compatibly as

$$
K_{m^{\prime}}=\left[\begin{array}{l}
K_{1} \\
K_{2}
\end{array}\right]\left\lceil m^{\prime}-m^{\prime}\right.
$$

then

$$
\begin{aligned}
\tilde{A}_{11}-A_{122} K \tilde{C}_{1} & =\tilde{A}_{11}-\left[\tilde{B}_{1} 0\right] K_{m^{\prime}} \tilde{C}_{1} \\
& =\tilde{A}_{11}-\tilde{B}_{1} K_{1} \tilde{C}_{1}
\end{aligned}
$$

and $\left(\tilde{A}_{11}, A_{122}, \tilde{C}_{1}\right)$ is stabilizable by output feedback if and only if $\left(\tilde{A}_{11}, \tilde{B}_{1}, \tilde{C}_{1}\right)$ is stabilizable by output feedback. The triple must be controllable, observable and satisfy the Kimura-Davison conditions, which yield

$$
m^{\prime}+\tilde{p}+r \geq n+1
$$

Lemma 4. [16] The pair $\left(\tilde{A}_{11}, \tilde{B}_{1}\right)$ is completely controllable and $\left(\tilde{A}_{11}, \tilde{C}_{1}\right)$ is completely observable.

The next subsection considers the problem of constructing an appropriate control law, based on the extended outputs only, to induce sliding.

\subsection{The Reachability Problem}

It will be shown that a sliding motion can be induced on the manifold $\{x \in$ $\left.\mathbb{R}^{n}: \tilde{F} \tilde{C} x=0\right\}$ using a higher order sliding mode method (see [2], [24], or 
[33] for further details). In order to stabilize the state of the system (1-2), the following output feedback controller is proposed:

$$
u=(\tilde{F} \tilde{C} B)^{-1}\left(-\Gamma \tilde{F} \tilde{y}-w_{\tilde{y}}\right)
$$

where $\Gamma$ is a strictly positive diagonal matrix and the auxiliary output $\tilde{y}$ is the output of the observer defined in $\S 3$.

$w_{\tilde{y}}$ is the super twisting algorithm defined componentwise by

$$
\left\{\begin{array}{l}
\left(w_{\tilde{y}}\right)_{i}=\varphi\left((\tilde{F} \tilde{y})_{i}\right)+\lambda_{i}\left|(\tilde{F} \tilde{y})_{i}\right|^{\frac{1}{2}} \operatorname{sign}\left((\tilde{F} \tilde{y})_{i}\right) \\
\dot{\varphi}\left((\tilde{F} \tilde{y})_{i}\right)=\alpha_{i} \operatorname{sign}\left((\tilde{F} \tilde{y})_{i}\right)
\end{array}\right.
$$

where $\lambda_{i}$ and $\alpha_{i}$ for $i=1, \ldots, m$ are strictly positive constants. This algorithm has been developed for systems with relative degree 1 to avoid chattering phenomena. The control law is made of two continuous terms. The discontinuity only appears in the control input time derivative. Note that $\tilde{y}$ only depends on the output of the system and that the components of $\tilde{y}$ are smooth functions with discontinuous time derivatives.

The first time derivative of $s_{e}$ is given by:

$$
\begin{aligned}
\dot{s}_{e} & =\tilde{F} \tilde{C}(A x(t)+B u(t)+f(t, x, u)) \\
& =\tilde{F} \tilde{C} A x(t)+\tilde{F} \tilde{C} f(t, x, u)-\Gamma F \tilde{y}-w_{\tilde{y}}
\end{aligned}
$$

Because after a finite time $T, \tilde{y}=\tilde{C} x$, one has:

$$
\dot{s}_{e}=\tilde{F} \tilde{C} A x(t)+\tilde{F} \tilde{C} f(t, x, u)-\Gamma s_{e}-w_{\tilde{y}}
$$

with

$$
\left\{\begin{array}{l}
\left(w_{\tilde{y}}\right)_{i}=\varphi\left(\left(s_{e}\right)_{i}\right)+\lambda_{i}\left|\left(s_{e}\right)_{i}\right|^{\frac{1}{2}} \operatorname{sign}\left(\left(s_{e}\right)_{i}\right) \\
\dot{\varphi}\left(\left(s_{e}\right)_{i}\right)=\alpha_{i} \operatorname{sign}\left(\left(s_{e}\right)_{i}\right)
\end{array}\right.
$$

for $i=1, \ldots, m$. The second time derivative $s_{e}$ is given by:

$$
\ddot{s}_{e}=\tilde{F} \tilde{C} A(A x(t)+B u(t)+f(\cdot))+\tilde{F} \tilde{C} \dot{f}(\cdot)-\Gamma \dot{s}_{e}-\dot{w}_{\tilde{y}}
$$

If the term $\|\tilde{F} \tilde{C} A(A x(t)+B u(t)+f(\cdot))+\tilde{F} \tilde{C} \dot{f}(\cdot)\|$ is bounded and if the control gains satisfy the conditions given in [31], finite time convergence on the sliding surface $\left\{s_{e}=\dot{s}_{e}=0\right\}$ is obtained. The system is asymptotically stabilized, because the sliding motion on $s_{e}=0$ has a stable dynamics.

\section{The sliding mode observer framework}

The scheme described in this section will be based on a classical observer of the form (5) for the system $\{A, D, \tilde{C}\}$. Consequently this requires (in realtime) the outputs that correspond to $\tilde{C} x$ from knowledge of only $y=C x$. In 
order to estimate the state of the system (1) with output (7), the following sliding mode observer is proposed:

$$
\dot{z}=A z+B u+G_{l}(\tilde{y}-\tilde{C} z)+G_{n} v_{c}
$$

The discontinuous output injection $v_{c}$ from (22) is defined by:

$$
v_{c}=\left\{\begin{array}{cl}
\rho \frac{P_{2}(\tilde{y}-\tilde{C} z)}{\left\|P_{2}(\tilde{y}-\tilde{C} z)\right\|} & \text { if }(\tilde{y}-\tilde{C} z) \neq 0 \\
0 & \text { otherwise }
\end{array}\right.
$$

where $\rho$ is a positive constant larger than the upper bound of $w$. The definition of the symmetric positive definite matrix $P_{2}$ can be found in [15] or in Chapter 6 of [17]. Still, $\tilde{y}$ is the output of the observer defined defined in $\S 3$.

Define the state estimation error $e=x-z$. Then it is straightforward to show that:

$$
\dot{e}=A e+D \xi-G_{l}(\tilde{y}-\tilde{C} z)-G_{n} v_{c}
$$

After a finite time $T, \tilde{y}=\tilde{C} x$ and for all $t>T$, the error dynamics (24) are given by:

$$
\dot{e}=\left(A-G_{l} \tilde{C}\right) e+D \xi-G_{n} v_{c}
$$

with

$$
v_{c}=\left\{\begin{array}{cl}
\rho \frac{P_{2}(\tilde{C} e)}{\left\|P_{2}(\tilde{C} e)\right\|} & \text { if }(\tilde{C} e) \neq 0 \\
0 & \text { otherwise }
\end{array}\right.
$$

Since by construction $\operatorname{rank}(\tilde{C} D)=\operatorname{rank}(D)$ and by assumption the invariant zeros of the triple $(A, D, \tilde{C})$ lie in the left half plane, the design methodologies given in [15], [17] or [38] can be applied so that $e=0$ is an asymptotically stable equilibrium point of (25) and the dynamics are independent of $\xi$ once a sliding motion on the sliding manifold $\{e: s=\tilde{C} e=0\}$ has been attained.

In addition, the method enables estimation of the unknown inputs. Define $\left(v_{c}\right)_{e q}$ as the equivalent output error injection required to maintain the sliding motion in (25). During the sliding motion,

$$
\dot{s}=\tilde{C} \dot{e}=\tilde{C}\left(A-G_{l} \tilde{C}\right) e+\tilde{C} D \xi-\tilde{C} G_{n} v_{c}(\tilde{C} e)=0
$$

Since $e \rightarrow 0$ and using (25):

$$
\tilde{C} G_{n}\left(v_{c}\right)_{e q} \rightarrow \tilde{C} D \xi
$$

As $\tilde{C} D$ is full rank, an approximation $\hat{\xi}$ of $\xi$ can be obtained from $\left(v_{c}\right)_{e q}$ by:

$$
\hat{\xi}=\left((\tilde{C} D)^{T} \tilde{C} D\right)^{-1}(\tilde{C} D)^{T} \tilde{C} G_{n}\left(v_{c}\right)_{e q}
$$


Note that during the sliding motion, the effect of the linear feedback $G_{l} \tilde{C} e$ disappears since $\tilde{C} e \equiv 0$. However the inclusion of $G_{l}$ is involved with the proof of global convergence of $e$ to zero and the construction of a Lyapunov function [15]. It also means that prior to sliding, (24) can be viewed as having filtering properties since by construction $A-G_{l} C$ is stable.

Remark 1. There exist in the literature several sliding mode observers that do not require the relative degree condition (in $[1,12,13,23,25,31]$ ). All these works deal with finite time state estimation and only consider systems without invariant zeros. Except for [23] and [31], these papers do not consider unknown inputs affecting the system and focus on the estimation of the states.

\section{Speed and tension control in a web-transport system}

The control of winding systems for handling webbed material such as textiles, paper, polymers and metals is of great industrial interest. Independent control of the velocity and tension of the material in the face of time-varying parameter changes in the radius of the material on the winder and unwinder reels is required. A model of a three motor winding system is given in [3] as described below:

$$
\begin{aligned}
N \dot{x} & =A x+B u \\
y & =C x
\end{aligned}
$$

where

$$
x=\left[V_{1}, T_{1}, V_{2}, T_{2}, V_{3}, T_{3}, V_{4}, T_{4}, V_{5}\right]^{T}
$$

and $u=\left[u_{u}, u_{v}, u_{w}\right]^{T}$ and $y=\left[T_{u}, V_{3}, T_{w}\right]^{T}$. The control inputs are the torque control signals applied to three brushless motors driving the unwinder, the master tractor and the winder respectively. The output measurements are the web tensions at the unwinder and winder, $T_{u}$ and $T_{w}$, respectively, and the web velocity, $V_{3}$, measured at the master tractor. The states of the system are the corresponding tensions, $T_{i}$, and web velocities $V_{i}$ at various points across the process. The matrices $A, B, C$ and $N$ are given below:

$$
A=\left(\begin{array}{ccccccccc}
-f_{1} & R_{1}^{2} & 0 & 0 & 0 & 0 & 0 & 0 & 0 \\
-E_{0} & -V_{0} & E_{0} & 0 & 0 & 0 & 0 & 0 & 0 \\
0 & -R_{2}^{2} & -f_{2} & R_{2}^{2} & 0 & 0 & 0 & 0 & 0 \\
0 & V_{0} & -E_{0} & -V_{0} & E_{0} & 0 & 0 & 0 & 0 \\
0 & 0 & 0 & -R_{3}^{2} & -f_{3} & R_{3}^{2} & 0 & 0 & 0 \\
0 & 0 & 0 & V_{0} & -E_{0} & -V_{0} & E_{0} & 0 & 0 \\
0 & 0 & 0 & 0 & 0 & -R_{4}^{2} & E_{0} & 0 & 0 \\
0 & 0 & 0 & 0 & 0 & V_{0} & -E_{0} & -V_{0} & E_{0} \\
0 & 0 & 0 & 0 & 0 & 0 & 0 & -R_{5}^{2} & -f_{5}
\end{array}\right)
$$




$$
\begin{aligned}
& B=\left(\begin{array}{ccc}
K_{u} R_{1} & 0 & 0 \\
0 & 0 & 0 \\
0 & 0 & 0 \\
0 & 0 & 0 \\
0 & K_{t} R_{3} & 0 \\
0 & 0 & 0 \\
0 & 0 & 0 \\
0 & 0 & 0 \\
0 & 0 & K_{w} R_{5}
\end{array}\right) \\
& C=\left(\begin{array}{ccccccccc}
0 & 0.5 & 0 & 0.5 & 0 & 0 & 0 & 0 & 0 \\
0 & 0 & 0 & 0 & 1 & 0 & 0 & 0 & 0 \\
0 & 0 & 0 & 0 & 0 & 0.5 & 0 & 0.5 & 0
\end{array}\right) \\
& N=\operatorname{diag}\left\{J_{1}, L, J_{2}, L, J_{3}, L, J_{4}, L, J_{5}\right\}
\end{aligned}
$$

In the above matrices, $V_{i}, R_{i}, J_{i}$ and $f_{i}$ are the linear velocity, the radius, the inertia and the viscous friction coefficient of the $i$ th roll, $L$ is the web length between two successive rolls, and $K_{u}, K_{t}$ and $K_{w}$ are the torque constants of the three motors. $V_{0}$ and $E_{0}$ are the nominal values of the linear web velocity and the elastic modulus of the material respectively. The nominal data values used to construct a linear model at start-up are taken from [29] and reported in Table 1.

\begin{tabular}{|c|c|c||c|c|c|}
\hline Notation & Value & Units & Notation & Value & Units \\
\hline$L$ & 0.45 & $\mathrm{~m}$ & $J_{2}$ & 0.00109 & $\mathrm{~kg} \cdot \mathrm{m}^{2}$ \\
\hline$V_{0}$ & $100 / 60$ & $\mathrm{~m} . \mathrm{s}^{-1}$ & $J_{3}$ & 0.00184 & $\mathrm{~kg} \cdot \mathrm{m}^{2}$ \\
\hline$E_{0}$ & 4175 & $\mathrm{~N} . \mathrm{m}$ & $J_{4}$ & 0.00109 & $\mathrm{~kg} . \mathrm{m}^{2}$ \\
\hline$R_{1}$ & 0.031 & $\mathrm{~m}$ & $J_{5}$ & 0.00109 & $\mathrm{~kg} \cdot \mathrm{m}^{2}$ \\
\hline$R_{2}$ & 0.02 & $\mathrm{~m}$ & $f_{1}$ & 0.0195 & N.m.s.rad \\
\hline$R_{3}$ & 0.035 & $\mathrm{~m}$ & $f_{2}$ & 0.000137 & N.m.s.rad \\
\hline$R_{4}$ & 0.02 & $\mathrm{~m}$ & $f_{3}$ & 0.0075 & N.m.s.rad \\
\hline$R_{5}$ & 0.032 & $\mathrm{~m}$ & $f_{4}$ & 0.000466 & N.m.s.rad \\
\hline$J_{1}$ & 0.0083 & $\mathrm{~kg} . \mathrm{m}^{2}$ & $f_{5}$ & 0.0045 & N.m.s.rad \\
\hline
\end{tabular}

Table 1. Parameters of the winding machine

Consider first the problem of output feedback control. For simplicity and without loss of generality, it is assumed that the system (27) is not subject to disturbances $f$. Note that $\operatorname{det}\left(C N^{-1} B\right)=0$ and so standard approaches for the design of sliding mode controllers based on output measurements cannot be applied to this system. The nominal system also possesses four transmission zeros located at $-3.7500 \pm 86.0751 i$ and $-2.1500 \pm 96.3089 i$. The winding system is thus seen to represent an appropriate case study to illustrate the work presented in this chapter. Define: 


$$
\tilde{C}=\left[\begin{array}{c}
C_{1} \\
C_{1} N^{-1} A \\
C_{2} \\
C_{3} \\
C_{3} N^{-1} A
\end{array}\right]=\left[\begin{array}{ccccccccc}
0 & \frac{1}{2} & 0 & \frac{1}{2} & 0 & 0 & 0 & 0 & 0 \\
-\frac{E_{0}}{2 L} & 0 & 0 & -\frac{V_{0}}{2 L} & \frac{E_{0}}{2 L} & 0 & 0 & 0 & 0 \\
0 & 0 & 0 & 0 & 1 & 0 & 0 & 0 & 0 \\
0 & 0 & 0 & 0 & 0 & \frac{1}{2} & 0 & \frac{1}{2} & 0 \\
0 & 0 & 0 & \frac{V_{0}}{2 L} & -\frac{E_{0}}{2 L} & 0 & 0 & -\frac{V_{0}}{2 L} & \frac{E_{0}}{2 L}
\end{array}\right]
$$

Then it is easy to verify $\tilde{C} B$ has full rank. Thus, the extended output is given by

$$
\tilde{y}=\left[\begin{array}{c}
y_{1} \\
\nu\left(y_{1}-\hat{y}_{1}\right) \\
y_{2} \\
y_{3} \\
\nu\left(y_{3}-\hat{y}_{3}\right)
\end{array}\right]
$$

and second and fifth outputs in $\tilde{y}$ are produced from (in this case) the degenerate step-by-step observers

$$
\begin{aligned}
& \dot{\hat{y}}_{1}=\nu\left(y_{1}-\hat{y}_{1}\right)+C_{1} N^{-1} B u \\
& \dot{\hat{y}}_{3}=\nu\left(y_{3}-\hat{y}_{3}\right)+C_{3} N^{-1} B u
\end{aligned}
$$

where $\nu$ is defined by (11).

As tracking of desired values for the web tensions at the unwinder and winder, $T_{u}$ and $T_{w}$, respectively, and the web velocity, $V_{3}$, is desired, an integral action methodology is employed. Define additional integral action states as

$$
\dot{\eta}=-C x+\eta y_{r e f}
$$

Note that the integral action states are defined for the measured plant outputs, and not the extended output. For the design of the sliding surface, the output matrix is defined in terms of the augmented state $[x, \eta]^{T}$ and given by

$$
\left[\begin{array}{cc}
\tilde{C} & 0 \\
0 & I_{3}
\end{array}\right]
$$

With this augmented output, the introduction of integral action produces no additional transmission zeros and the design procedure follows that described earlier. With 12 states, 3 inputs and 8 effective outputs, the reduced order stabilization problem defining the switching surface dynamics is a state feedback problem and the 5 poles available for selection are placed at $-0.75,-2$, $-0.5,-1$ and -1.5 . These poles, together with the transmission zeros of the original system, wholly determine the dynamics in the sliding mode. The extended outputs are formed by two differentiators, each with gains $l_{1}=1000$ and $l_{2}=500$. The parameters of the twisting algorithm are given by $\alpha_{1}=2$, $\lambda_{1}=1, \alpha_{2}=200, \lambda_{2}=100, \alpha_{3}=200$ and $\lambda_{3}=50$. Under nominal operating conditions, where fixed parameter values are used and no nonlinearity is present, the system was required to track a velocity offset command of $1 \mathrm{~m} / \mathrm{s}$ and desired offset tensions of $0.1 \mathrm{~N}$. After 20 seconds, a further offset of $0.5 \mathrm{~N}$ 


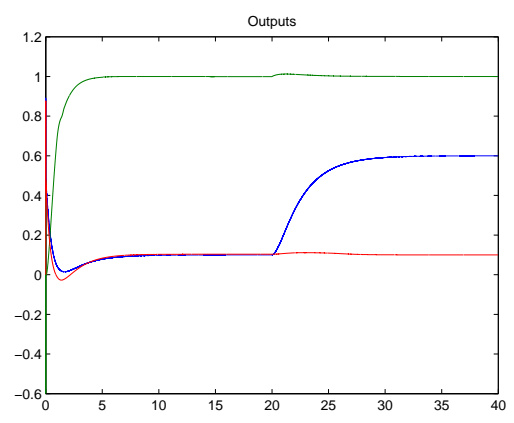

Fig. 1. Evolution of the outputs for the nominal linear plant model

was demanded at the winder. As can be seen from Figure 1, the set point changes are accurately attained with minimal coupling between the outputs.

In Figure 2, the evolution of the outputs is shown for the same reference demands, but this time in the presence of variations in the radii of the rolls. The system starts with a diameter of $0.15 \mathrm{~m}$ of material on the unwinder. During the nonlinear simulation, the material is wound onto the winder. The simulation incorporates parameter changes and the nonlinear behaviour of the radii. Visually, the results are seen to be very similar. The robustness of the method is thus highlighted.

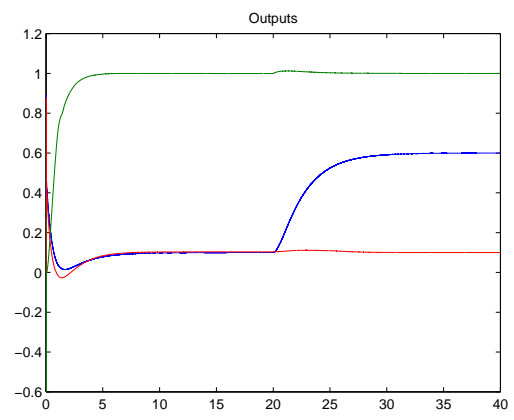

Fig. 2. Evolution of the outputs for the nonlinear, time varying plant

For the observer design problem, consider the same three motor winding systems with unknown inputs:

$$
\begin{aligned}
N \dot{x} & =A x+B u+D \xi \\
y & =C x
\end{aligned}
$$


The signal $\xi=\left[\xi_{1}(t) \xi_{2}(t) \xi_{3}(t)\right]^{T}$ represents the unknown inputs vector and it is assumed the unknown input distribution matrix is given by $D=B$. Thus, the bounded signal $\xi_{i}$ may represent an actuator fault in such a way that $\xi_{i}(t) \neq 0$ when a fault appears and is zero in the fault free case. Since $\operatorname{det}\left(C N^{-1} D\right)=0$, standard sliding mode observer and other UIO approaches cannot be applied to this system but the augmented output used for the control problem may be employed. Consequently using the ideas in $\S 5$, the following 'classical' sliding mode observer can be designed:

$$
\dot{z}=N^{-1} A z+N^{-1} B u+G_{l}(\tilde{y}-\tilde{C} z)+G_{n} v_{c}
$$

where $v_{c}$ is the discontinuous (unit vector) output injection term as in (23), and $\tilde{y}$ and $\tilde{C}$ are given by (34) and (33), respectively. Define the observation errors as $e=x-z$ and $e_{y_{1}}=y_{1}-\hat{y}_{1}, e_{y_{3}}=y_{3}-\hat{y}_{3}$. Then the error dynamics are given by:

$$
\begin{aligned}
\dot{e} & =N^{-1} A e+N^{-1} D \xi-G_{l}(\tilde{y}-\tilde{C} z)-G_{n} v_{c} \\
\dot{e}_{y_{1}} & =C_{1}\left(N^{-1} A x+N^{-1} B u+N^{-1} D \xi\right)-\nu\left(e_{y_{1}}\right)-C_{1} N^{-1} B u \\
& =C_{1} N^{-1} A x-\nu\left(e_{y_{1}}\right) \\
\dot{e}_{y_{3}} & =C_{3}\left(N^{-1} A x+N^{-1} B u+N^{-1} D \xi\right)-\nu\left(e_{y_{3}}\right)-C_{3} N^{-1} B u \\
& =C_{3} N^{-1} A x-\nu\left(e_{y_{3}}\right)
\end{aligned}
$$

As in [31], choose $\lambda_{s}$ and $\alpha_{s}$ large enough such that after a finite time $T_{i}$, $e_{y_{i}}=\dot{e}_{y_{i}}=0$, and $\nu\left(e_{y_{i}}\right)=C_{i} N^{-1} A x, i=1,3$. This implies that for $t>$ $\max \left\{T_{1}, T_{3}\right\}$, system (39)-(41) becomes:

$$
\begin{aligned}
\dot{e} & =\left(N^{-1} A-G_{l} \tilde{C}\right) e+N^{-1} D \xi-G_{n} v_{c} \\
\dot{e}_{y_{1}} & =\dot{e}_{y_{3}}=0
\end{aligned}
$$

In the simulations, the following observer parameters have been chosen. The two scalar gains associated with the observers to estimate $\dot{y}_{1}$ and $\dot{y}_{3}$ are $\lambda_{s}=$ 300 and $\alpha_{s}=8000$. The scalar gain associated with the first order sliding mode discontinuous injection $v_{c}$ is $\rho=1.5$. The control signal $u$ has been set to zero without loss of generality. The unknown inputs have been chosen as follows: $\xi_{1}$ is a square wave of amplitude 0.1 and frequency $0.1 \mathrm{~Hz}$ that starts at $t=5 \mathrm{~s} ; \xi_{2}$ is a sine wave of amplitude 0.2 and frequency $1 \mathrm{~Hz}$ that starts at $t=0 \mathrm{~s} ; \xi_{3}$ is a sawtooth signal of amplitude 0.05 and frequency $0.4 \mathrm{~Hz}$ that starts at $t=0 \mathrm{~s}$. Note that at a finite set of isolated points in time, the derivatives of the unknown input signals do not exist. However the differential equations are satisfied almost everywhere. Figures 3 and 4 show that the state is accurately estimated in spite of the three actuator faults. It can be seen in Figure 5 that the unknown input signals are also accurately reconstructed. 
A simulation has been made with a $10 \%$ variation of the viscous coefficient $f_{2}$. Again, all states were recovered as well as the three unknown inputs. This is shown in Figure 6. Another simulation for testing robustness issue has been realized by considering a $20 \%$ variation of Young modulus $E_{0}$. The results of the unknown input reconstruction are shown in Figure 7. The numerical results indicate that the actuator fault detection scheme is tractable even with parameter uncertainties. This is important for instance if several materials with different Youngs modulus have to be used on the same winding machine.

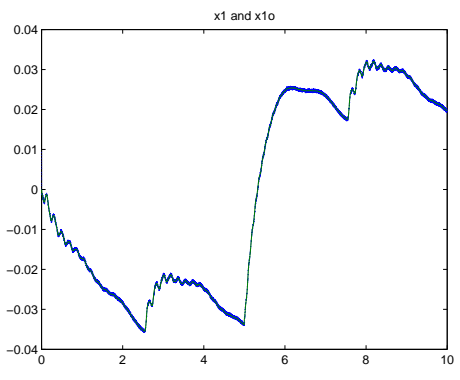

(a)

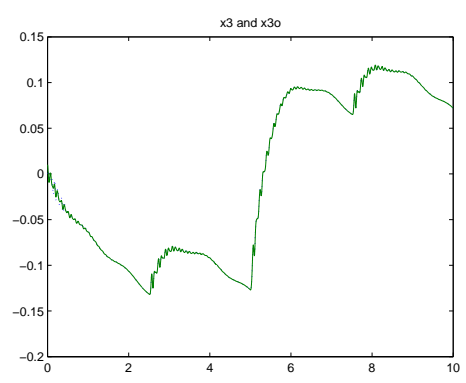

(c)

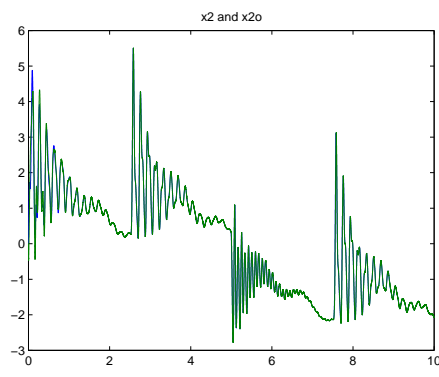

(b)

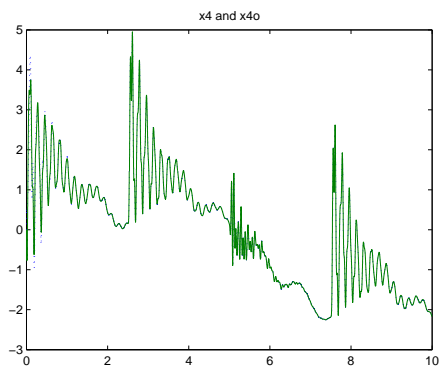

(d)

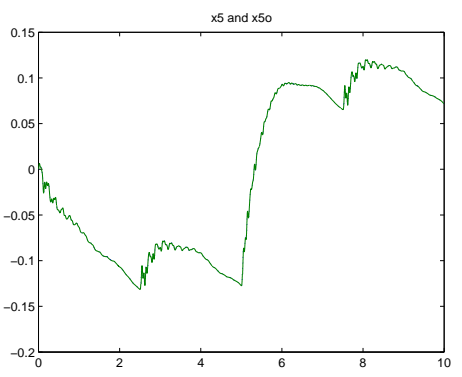

(e)

Fig. 3. State and estimation (nominal case) 


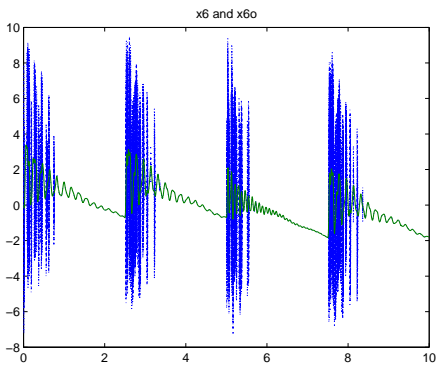

(a)

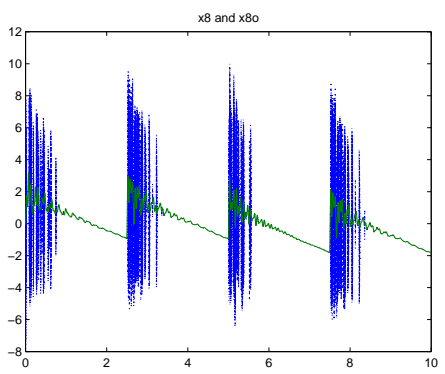

(c)

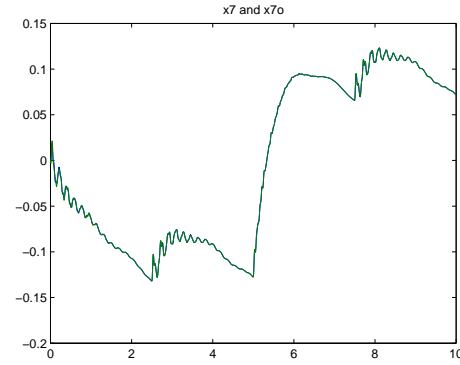

(b)

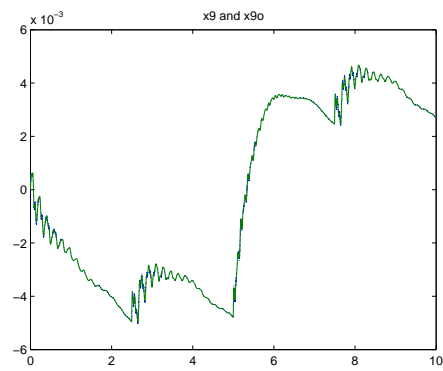

(d)

Fig. 4. State and estimation (nominal case)

\section{Concluding remarks}

This chapter has presented both an output feedback sliding mode control design framework and a sliding mode observer design approach for MIMO systems of arbitrary relative degree. A minimal set of outputs and output derivatives have been identified to determine an augmented system which is relative degree one, and a robust sliding mode differentiator has been used as a mechanism to construct the extended output signal. It has been shown that the transmission zeros of the original triple appear directly in the reduced order sliding mode dynamics relating to the augmented system. For the static output feedback control problem a super twisting control algorithm has been shown to provide robust control performance. The problem of designing a sliding mode unknown input observer for linear systems has been broadened using the same approach. The scheme is based on a 'classical' sliding mode observer used in conjunction with a scheme to estimate a certain number of derivatives of the outputs. The number of derivatives required is system dependent and can be easily calculated. By using the equivalent output injections from the derivative estimation scheme and the classical observer, estimation of both 


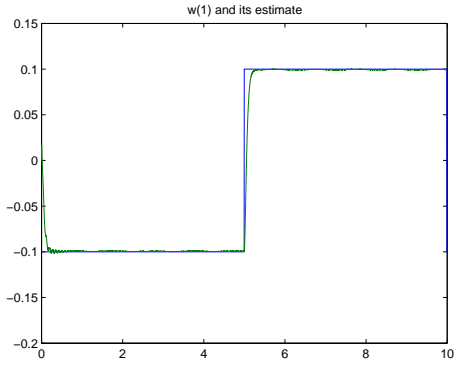

(a)

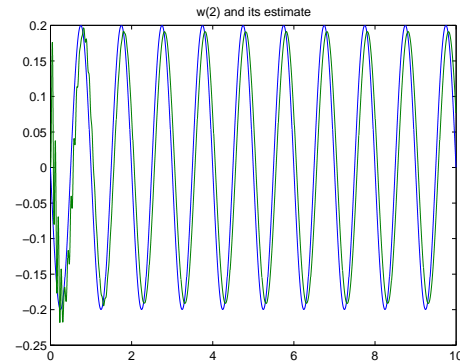

(b)

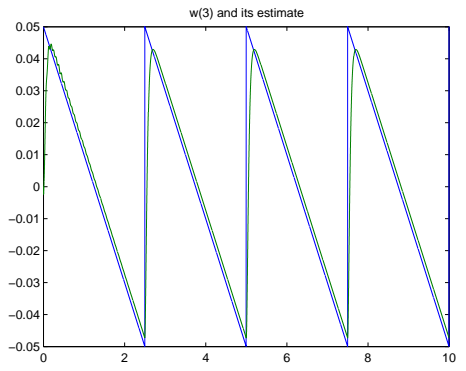

(c)

Fig. 5. Unknown input and estimation (nominal case)

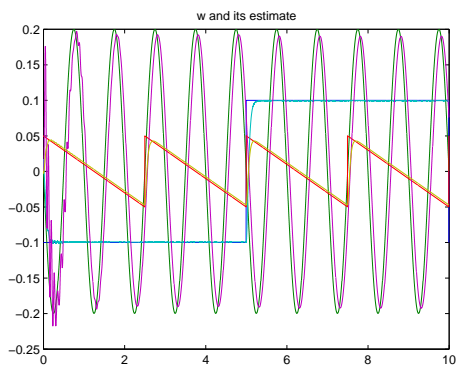

Fig. 6. Unknown input and estimation: $10 \%$ variation of the viscous coefficient $f_{2}$

the system state and the unknown inputs can be obtained. Since the derivative estimation observer is based on second order sliding mode algorithms, the equivalent output injections are obtained in a continuous way without the use of low pass filters. 


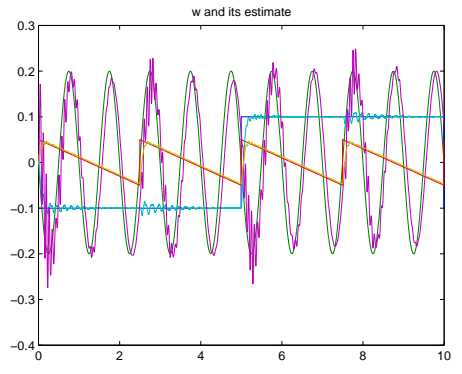

Fig. 7. Unknown input and estimation: $20 \%$ variation of Young modulus $E_{0}$

\section{References}

1. J.-P. Barbot, T. Boukhobza and M. Djemaï, "Sliding mode observer for triangular input form", IEEE Conf. on Decision and Control, Japan, 1996.

2. Bartolini, G., Ferrara, A., Levant, A., \& Usai, E. (1999). "On second order sliding mode controllers". Variable structure systems, sliding mode and nonlinear control, K.D. Young and Ü. Özgüner Eds., Lecture Notes in Control and Information Sciences 247, Springer-Verlag, London, 329-350.

3. A. Benlatreche, D. Knittel and E. Ostertag, "Robust decentralised control strategies for large-scale web handling systems", Control Engineering Practice, to appear, available online at www.sciencedirect.com.

4. C. Canudas de Wit and J. J. E. Slotine, "Sliding observers in robot manipulators", Automatica, Vol. 27, No 5, pp. 859-864, 1991.

5. C.L.Chen, K.C.Lin and M.J.Jang, "On chattering free output feedback sliding mode design for MIMO linear systems", Proceedings of the 16th IFAC World Congress, Prague, July 3-8, 2005.

6. J. Chen, R. Patton, and H. Zhang. "Design of unknown input observers and robust fault detection filters", International Journal of Control, 63:85-105, 1996.

7. J. Chen and H. Zhang, "Robust detection of faulty actuators via unknown input observers", International Journal of Systems Science, 22:1829-1839, 1991.

8. M. Corless and J. Tu, "State and input estimation for a class of uncertain systems", Automatica, Vol. 34, No 6, pp. 757-764, 1998.

9. M. Darouach, "On the novel approach to the design of unknown input observers", IEEE Transactions on Automatic Control, 39:698-699, 1994.

10. M. Darouach, M. Zasadzinski, and S. J. Xu, "Full-order observers for linear systems with unknown inputs", IEEE Transactions on Automatic Control, 39:606609, 1994.

11. M. Djemaï, J.-P. Barbot, A. Glumineau and R. Boisliveau, "Nonlinear flux sliding mode observer", in IEEE CSCC99, IMACS, Athens, Greece, 1999.

12. S. V. Drakunov, "Sliding-mode Observer Based on Equivalent Control Method", in IEEE Conf. on Decision and Control, Tucson, Arizona, 1992.

13. S. V. Drakunov and V. I. Utkin, "Sliding mode observers. Tutorial", in IEEE Conference on Decision and Control, New-Orleans, LA, 1995.

14. B. Drazenovic, "The Invariance Conditions in Variable Structure Systems", $A u$ tomatica, Vol. 5, No 3, pp. 287-295, 1969. 
15. C. Edwards and S. K. Spurgeon, "On the development of discontinuous observers", International Journal of Control, Vol. 59, pp. 1211-1229, 1994.

16. C. Edwards, and S.K. Spurgeon, "Sliding mode stabilisation of uncertain systems using only output information", International Journal of Control, Vol. 62, pp. 1129-1144, 1995.

17. C. Edwards and S. K. Spurgeon, Sliding mode control: theory and applications, Taylor and Francis Eds, 1998.

18. C. Edwards and S. K. Spurgeon and R. J. Patton, "Sliding mode observers for fault detection and isolation", Automatica, Vol. 36, pp. 541-553, 2000.

19. C. Edwards, "A comparison of sliding mode and unknown input observers for fault reconstruction", in IEEE Conf. on Decision and Control, Bahamas, 2004.

20. C. Edwards and C. P. Tan, "Sensor fault tolerant control using sliding mode observers", Control Engineering Practice, , Vol. 14, No 8, Pages 897-908, 2006.

21. T. Floquet, J.P. Barbot, W. Perruquetti, "A finite time observer for flux estimation in the induction machine", Conference on Control Applications, Glasgow, Scotland, 2002.

22. T. Floquet, J.-P. Barbot, W. Perruquetti and M. Djemaï, "On the robust fault detection via a sliding mode disturbance observer", International Journal of Control, Vol. 77, No 7, pp. 622-629, 2004.

23. T. Floquet and J.P. Barbot, "A canonical form for the design of unknown input sliding mode observers", in Advances in Variable Structure and Sliding Mode Control, Lecture Notes in Control and Information Sciences, Vol. 334, C. Edwards, E. Fossas Colet, L. Fridman, (Eds.), Springer Edition, 2006.

24. L. Fridman and A. Levant, "Higher order sliding modes", in W. Perruquetti and J. P. Barbot (Eds), Sliding Mode Control in Engineering, Marcel Dekker, pp. 53-101, 2002.

25. I. Haskara, Ü. Özgüner and V. I. Utkin, "On sliding mode observers via equivalent control approach", International Journal of control, Vol. 71, No. 6, pp. 1051-1067, 1998.

26. M. L. J. Hautus, "Strong detectability and observers", Linear Algebra and its Applications, Vol. 50, pp. 353-368 1983.

27. J. Hernandez et J.-P. Barbot, "Sliding observer-based feedback control for flexible joints manipulator", Automatica, Vol. 32, No 9, pp. 1243-1254, 1996.

28. M. Hou and P. C. Müller, "Design of observers for linear systems with unknown inputs", IEEE Transactions on Automatic Control, Vol. 37, No. 6, pp. 871-875, 1992.

29. H. Koc, D. Knittel, M. de Mathelin, G. Abba, "Modeling and robust control of winding systems for elastic webs", IEEE Transactions on Control Systems Technology, Vol. 10, No 2, pp. 197-208, 2002.

30. C.M. Kwan, "Further results on variable output feedback controllers", IEEE Transactions on Automatic Control, Vol. 46, pp. 1505-1508, 2001.

31. A. Levant, "Robust exact differentiation via sliding mode technique", Automatica, Vol. 34, No. 3, pp. 379-384, 1998.

32. W. Perruquetti, T. Floquet, P. Borne, "A note on sliding observer and controller for generalized canonical forms", IEEE Conf. on Decision and Control, Tampa, Florida, USA, 1998.

33. W. Perruquetti and J.-P. Barbot (Editors), Sliding Mode Control in Engineering, Marcel Dekker, 2002. 
34. H. Saadaoui, N. Manamanni, M. Djemaï, J.-P. Barbot and T. Floquet, "Exact differentiation and sliding mode observers for switched Lagrangian systems", Nonlinear Analysis Theory, Methods \& Applications, Vol. 65, No 5, pp 10501069, 2006.

35. K.K. Shyu, Y.W. Tsai and C.K. Lai, "A dynamic output feedback controller for mismatched uncertain variable structure systems", Automatica, Vol. 37, pp. $775-779,2001$.

36. J. J. Slotine, J. K. Hedrick, and E. A. Misawa, "On sliding observers for nonlinear systems", ASME J. Dyn. System Measurement Control, vol. 109, pp. 245-252, 1987.

37. H. J. Sussman and P. V. Kokotovic, "The peaking phenomenon and the global stabilization of nonlinear systems", IEEE Transactions on Automatic Control, Vol. 36, No 4, pp. 424-440, 1991.

38. C. P. Tan, and C. Edwards, "An LMI approach for designing sliding mode observers", International Journal of Control, Vol. 74(16), pp. 1559-1568, 2001.

39. C. P. Tan and C. Edwards, "Sliding mode observers for robust detection and reconstruction of actuator and sensor faults", Int. J. of robust and nonlinear control, Vol. 13, pp. 443-463, 2003.

40. V. I. Utkin, Sliding Modes in Control and Optimization, Berlin, Germany, Springer-Verlag, 1992.

41. V. I. Utkin, J. Guldner and J. Shi, Sliding mode control in electromechanical systems, Taylor and Francis, London, 1999.

42. B. L. Walcott and S. H. Żak, "State observation of nonlinear uncertain dynamical systems", IEEE Transactions on Automatic Control, Vol. 32, pp. 166-170, 1987.

43. Y. Xiong and M. Saif, "Sliding Mode Observer for Nonlinear Uncertain Systems", IEEE Transactions on Automatic Control, vol. 46, pp. 2012-2017, 2001.

44. X.Y. Yan, S.K. Spurgeon and C. Edwards, "Dynamic Output Feedback Sliding Mode Control for a Class of Nonlinear Systems with Mismatched Uncertainty", European Journal of Control, Vol. 11, No. 1, pp. 1-10, 2005.

45. S.H. Zak, and S. Hui, "On variable structure output feedback controllers for uncertain dynamic" systems, IEEE Transactions on Automatic Control, Vol. 38, pp. $1509-1512,1993$ 